\title{
Early and late stroke after mitral valve replacement with a mechanical prosthesis: Risk factor analysis of a 24-year experience
}

Ko Bando, MD

Junjiro Kobayashi, $\mathrm{MD}^{\mathrm{a}}$

Mitsuhiro Hirata, $\mathrm{MD}^{\mathrm{a}}$

Toshihiko Satoh, MD, MPH

Kazuo Niwaya, MD

Osamu Tagusari, MDa

Satoshi Nakatani, $\mathrm{MD}^{\mathrm{b}}$

Toshikatsu Yagihara, MDa

Soichiro Kitamura, $\mathrm{MD}^{\mathrm{a}}$

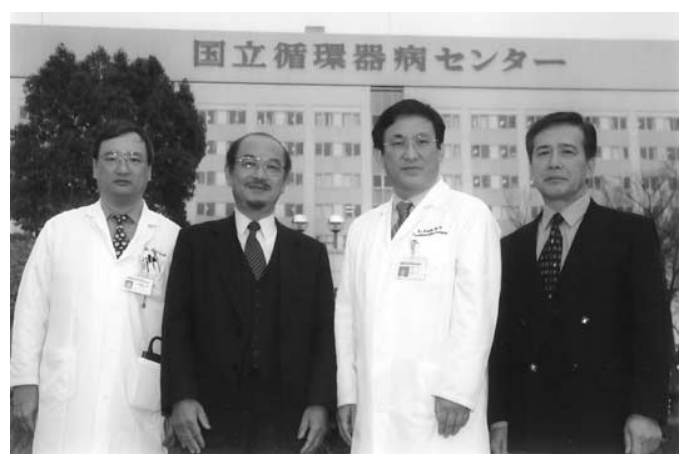

Kobayashi, Kitamura, Bando, Yagihara (left to right)
Objective: We evaluated risk factors for mortality and stroke after mechanical mitral valve replacement between May 1977 and December 2001.

Methods: Early and late mortality and stroke were assessed. Potential predictors of mortality and stroke were entered into a Cox proportional hazards model. Actuarial survival and freedom from stroke were determined by a log-rank test.

Results: Mitral valve replacement was performed in 812 patients. Concomitant procedures included left atrial appendage closure in $493(61 \%)$ patients, tricuspid annuloplasty-replacement in 348 (43\%) patients, maze procedure in 185 (23\%) patients, plication of the left atrium in $148(18 \%)$ patients, and other procedures in $151(19 \%)$ patients. Five-year actuarial survival was $91.1 \% \pm 2.3 \%$. Freedom from stroke at 8 years was significantly better in patients with sinus rhythm versus atrial fibrillation $(P<.001)$. Ninety-nine percent of patients with mitral valve replacement combined with a maze procedure were free from stroke, whereas only $89 \%$ of patients with mitral valve replacement alone were free from stroke at 8 years after surgical intervention. Seventy-two patients had late stroke; sixty-five patients (90\%) were in atrial fibrillation, and $47(65 \%)$ patients had the left atrial appendage closed. Multivariate analysis showed that late atrial fibrillation (odds ratio, 3.39; 95\% confidence interval, 1.72-6.67; $P=.0001$ ) and omission of the maze procedure (odds ratio, $3.40 ; 95 \%$ confidence interval, $1.14-10.14 ; P=.003$ ) were the significant risk factors for late stroke.

Conclusions: Persistent atrial fibrillation was the most significant risk factor for late stroke after mechanical mitral valve replacement. Restoration of sinus rhythm with a maze procedure nearly eliminated the risk of late stroke, whereas neither closure of the left atrial appendage nor therapeutic anticoagulation prevented this complication.

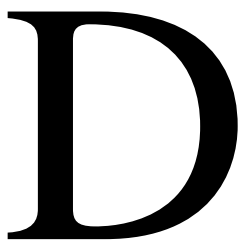

espite improvements in valve design, stroke remains a serious complication after heart valve replacement. It is generally agreed that lifelong anticoagulant therapy is indicated in all patients with mechanical valves. Recent studies indicate that major systemic embolism still occurs at a rate of $2 \%$ to $3 \%$ per year after mechanical mitral valve replacement (MVR), despite anticoagulation. ${ }^{1-3} \mathrm{We}$ and others recently demonstrated that maintaining sinus rhythm by 
using the Cox maze procedure reduced the incidence of late stroke. ${ }^{4-6}$ However, the risk for late stroke after mechanical valve replacement remains to be determined.

The purpose of the study was to elucidate the risks for early and late mortality and stroke after mechanical MVR in a single center over a 24-year period.

\section{Patients and Methods}

Between May 1977 and December 2001, we performed 812 MVRs with mechanical prostheses. We retrospectively reviewed the data from operative notes, anesthesia records, clinical case histories, and laboratory investigations, including electrocardiograms, echocardiograms, and cardiac catheterization reports. This retrospective study was approved by the Institutional Review Board of National Cardiovascular Center. Follow-up data were collected from the National Cardiovascular Center records of outpatient visits and correspondence with referring physicians. Each patient was followed by local physicians or by cardiologists or surgeons in our hospital at least every 2 months. A total of 33 clinical, hemodynamic, electrocardiographic, and echocardiographic variables were entered into a computerized database and analyzed. Follow-up data beyond 6 months after the operation were available for all patients.

\section{Definitions}

Stroke was defined as cerebral thromboembolism diagnosed by a neurologist and confirmed by means of a computed tomographic scan. Transient ischemic attacks were not counted as strokes in this study. Valve thrombosis was defined as impairment of the valve function by the deposition of thrombus demonstrated at operation or autopsy. Bleeding includes intracranial bleeding, spinal bleeding, and major extracranial bleeding. Intracranial and spinal bleeding were defined as a neurologic deficit of sudden or subacute onset confirmed by means of computed tomographic scanning, surgical investigation, or autopsy. Major extracranial bleeding was defined as an acute bleeding event that led to death or hospital admission or treatment of bleeding; bleeding that led to hospital admission for diagnostic procedures only was not considered major. Bleeding caused by trauma was also excluded. We also excluded all complications that occurred during hospitalization for a different reason because other diseases, interventions, and diagnostic procedures might affect the risk of complications far more than the intensity of the anticoagulant therapy., 7

\section{Patients}

Demographic data and preoperative cardiac information are given in Table 1. With respect to preoperative heart rhythm, 78\% of patients had chronic atrial fibrillation. In May 1992, the Cox maze procedure was introduced. Between May 1992 and December 1994, the Cox maze III procedure was the procedure primarily performed. A modified maze III procedure (Kosakai maze) was performed until May 1998. The cryo-maze procedure was used thereafter. Details of these procedures have been previously described. $^{6,9}$ Five hundred thirty-three $(66 \%)$ patients had St Jude Medical valves (St Jude Medical, Inc, St Paul, Minn). The remaining patients had a variety of valves including CarboMedics ( $\mathrm{n}=$ 125; Sulzer Carbomedics, Inc, Austin, Tex), Björk-Shiley ( $\mathrm{n}=70$,
TABLE 1. Preoperative clinical characteristics

\begin{tabular}{lc}
\hline Variable & MVR (n = 812) \\
\hline Sex (M/F) & $331 / 481$ \\
Age (range/median) & $18-79(58)$ \\
NYHA (class III/IV) No (\%) & $482(60 \%)$ \\
History of stroke No (\%) & $138(17 \%)$ \\
Left atrial thrombus No (\%) & $114(14 \%)$ \\
Previous cardiac surgery No (\%) & $369(45 \%)$ \\
Chronic AF No (\%) & $630(78 \%)$
\end{tabular}

NYHA, New York Heart Association; $A F$, atrial fibrillation.

in our early experience; Shiley, Inc, Irvine, Calif), ATS ( $n=47$; ATS Medical, Inc, Minneapolis, Minn), Omniscience ( $\mathrm{n}=29$; Medical Inc, Inver Grove Heights, Minn), and other valves ( $\mathrm{n}=$ 8). All valves were placed in the antianatomic position.

\section{Assessment of the Intensity of Anticoagulant Therapy}

The incidence of adverse events for specific levels of intensity of anticoagulation were calculated as the ratio of the number of events that took place when the prothrombin time was in a particular international normalized ratio (INR) range to the number of patient-years during which the INR was at this level in the patient population. This method has been described in detail previously. ${ }^{10}$

\section{Statistical Methods}

Survival and freedom from mortality, atrial fibrillation, and stroke were estimated by using the Kaplan-Meier method. Survivorship curves were compared with a log-rank test. The risks for death, recurrence of atrial fibrillation, and stroke were analyzed by using both univariate analysis and multivariate analysis with a Cox proportional hazards model.

\section{Results \\ Pathophysiology of Mitral Valve Disease and Operative Findings}

The pathophysiology of the mitral valve disease and the nature of concomitant procedures are depicted in Table 2. All patients underwent surgical intervention with mild hypothermia. Antegrade crystalloid cardioplegia was primarily used before 1987, and combined antegrade-retrograde blood cardioplegia was extensively used thereafter. Fiftyone percent (320/627) of the patients undergoing MVR alone underwent concomitant ligation of the left atrial appendage, whereas $94 \%(173 / 185)$ of the patients who underwent combined MVR and the maze procedure had the left atrial appendage closed.

\section{Postoperative Morbidity and Mortality}

Hospital death occurred in $33(4.1 \%)$ patients, and causes of deaths are listed in Table 3. Postoperative complications included bleeding $(n=14)$, renal failure $(n=6)$, intraaortic balloon pump insertion $(n=4)$, mediastinitis $(n=3)$, left ventricular assist device insertion $(n=3)$, left ventricular rupture $(n=3)$, and pneumothorax $(n=2)$. 
TABLE 2. Pathophisiology for mitral disease and concomitant procedure

\begin{tabular}{lc}
\hline Pathophisiology & MVR (n = 812) \\
\hline MS No. (\%) & $237(29 \%)$ \\
MR No. (\%) & $223(27 \%)$ \\
MSR No. (\%) & $169(21 \%)$ \\
PVF No. (\%) & $183(23 \%)$ \\
Concomitant procedure & \\
Maze procedure No (\%) & $185(23 \%)$ \\
LAA closure No. (\%) & $493(55 \%)$ \\
LAA plication No. (\%) & $148(18 \%)$ \\
Tricuspid valve surgery No (\%) & $348(43 \%)$ \\
TAP No. (\%) & $330(41 \%)$ \\
TVR No. (\%) & $18(2.2 \%)$ \\
CABG No. (\%) & $25(3 \%)$ \\
Others No. (\%) & $126(16 \%)$ \\
\hline
\end{tabular}

$M S$, Mitral stenosis; $M R$, mitral regurgitation; $M S R$, mitral stenosis/regurgitation; $P V F$, prosthetic valve failure; $L A A$, left atrial appendage; $T A P$, tricuspid annuloplasty; TVR, tricuspid valve replacement; $C A B G$, coronary artery bypass grafting

TABLE 3. Cause of early and late mortality

\begin{tabular}{llr}
\hline Early $33(4.1 \%)$ & LOS & 18 \\
& MOF & 7 \\
& Cerebral infarction & 3 \\
& Cerebral bleeding & 1 \\
Late $50(6.2 \%)$ & Others & 4 \\
& LOS & 10 \\
& MOF & 7 \\
& Cerebral infarction & 8 \\
& Cerebral bleeding & 7 \\
& Others & 18 \\
\hline
\end{tabular}

LOS, Low-output syndrome; MOF, multisystem organ failure.

TABLE 4. Results of univariate and multivariate analysis for late mortality

\begin{tabular}{lccccc}
\hline & Univariate & & \multicolumn{3}{c}{ Multivariate } \\
\cline { 2 - 3 } \cline { 5 - 6 } Variable & $\boldsymbol{P}$ value & & $\boldsymbol{P}$ value & Hazard ratio & $\mathbf{9 5 \%} \mathbf{~ C l}$ \\
\hline $\begin{array}{l}\text { Preoperative } \\
\quad \text { NYHA class IV }\end{array}$ & .0090 & .0032 & 3.39 & $1.50-7.65$ \\
Age $>65$ y & .0059 & & .0001 & 2.71 & $1.50-4.92$
\end{tabular}

$\mathrm{Cl}$, Confidence interval; NYHA, New York Heart Association.

\section{Survival and Late Mortality}

All patients were observed for at least 6 months after their operations. Actuarial 5-year, 10-year, and 15-year survivals were $91.1 \% \pm 1.8 \%, 88.4 \% \pm 2.3 \%$, and $85.4 \pm 3.2 \%$, respectively (Figure 1). There were $50(6.2 \%)$ late deaths. The causes of late deaths are listed in Table 3. Risk factors for late mortality are depicted in Table 4.

\section{Recurrence of Atrial Fibrillation}

We chose to analyze recurrence of arrhythmia after the first 30 days because early postoperative atrial fibrillation might

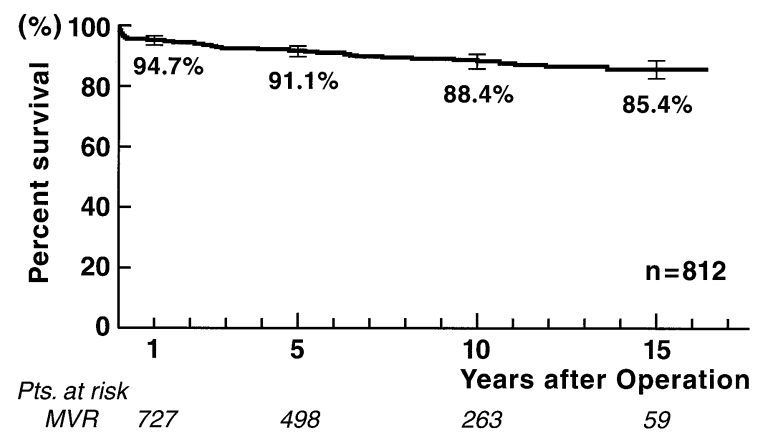

Figure 1. Actuarial survival curve. Bar indicates $95 \%$ confidence interval.

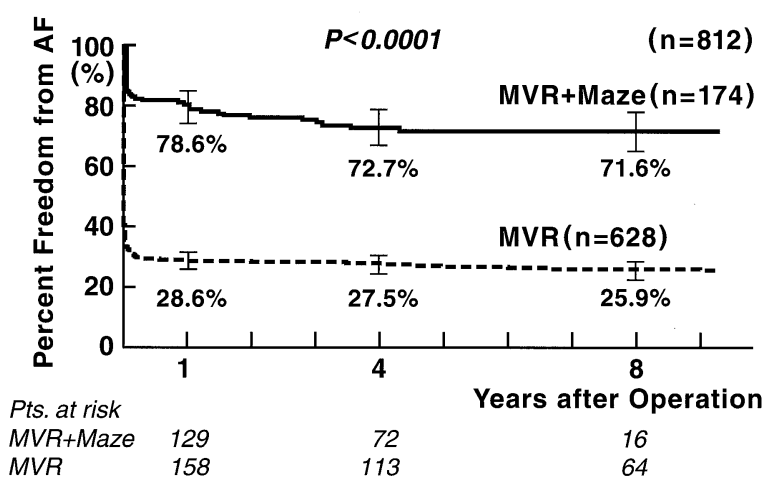

Figure 2. Freedom from recurrence of atrial fibrillation (AF). Bar indicates $95 \%$ confidence interval. MVR, Mitral valve replacement.

be caused by different mechanisms than those of chronic atrial fibrillation. Freedom from atrial fibrillation in the group undergoing MVR alone at 8 years was $25.9 \% \pm 3.2 \%$ compared with $71.6 \% \pm 6.3 \%$ in the combined MVR and maze group $(P<.0001$, Figure 2$)$. The incidence of stroke was similar between the patients with sinus rhythm after a maze procedure and those in native sinus rhythm (Appendix 1). Moreover, 46 patients with a combined MVR and maze procedure had a recurrence of atrial fibrillation late after surgical intervention.

\section{Preoperative and Postoperative Medications}

The spectrum and intensity of preoperative antiarrhythmic drugs were similar in patients with and without a concomitant maze procedure. The number of antiarrhythmic agents decreased over time in the combined maze-MVR group, whereas the vast majority of patients undergoing MVR alone continued to receive $\beta$-blockers and digoxin for rate control. All patients were taking warfarin, and the target INR was 1.8 to $2.8 .^{11,12}$ Only 27 patients took antiplatelet agents; this did not affect the statistical results. 


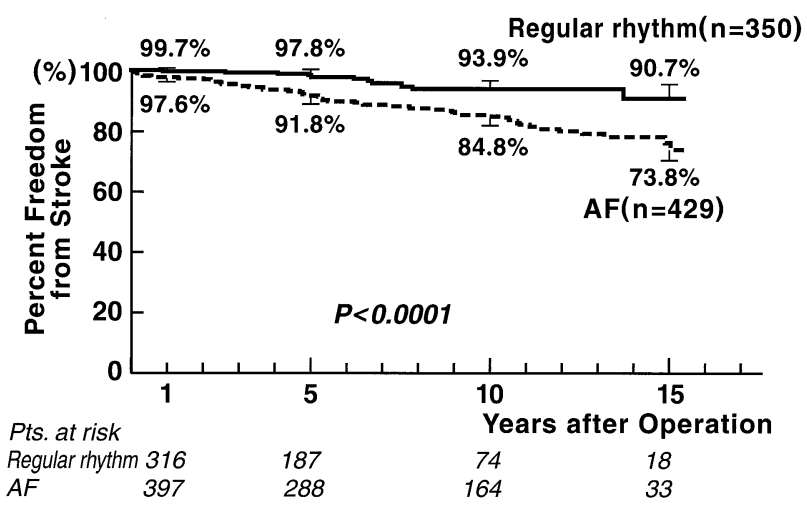

Figure 3. Freedom from stroke stratified by rhythm at 1 month after surgical intervention. Bar indicates $95 \%$ confidence interval. $A F$, Atrial fibrillation.

\section{Incidence of Stroke After Surgical Intervention}

Seventy-two patients had a late stroke; $65(90 \%)$ patients were in atrial fibrillation, and $47(65 \%)$ patients had the left atrial appendage closed. Irrespective of preoperative rhythm (either in sinus rhythm or atrial fibrillation), closure of the left atrial appendage did not have a significant effect on the incidence of stroke, even in patients undergoing MVR alone. At the time of their stroke, 63 (86\%) patients had an INR of greater than 1.8. Nine patients had stopped warfarin for minor operations or dental care; their INR was less than 1.5.

Freedom from stroke 15 years after surgical intervention was $90.7 \% \pm 6.5 \%$ in patients with regular rhythm compared with $73.8 \% \pm 6.9 \%$ in patients with chronic atrial fibrillation $(P<.0001$, Figure 3$)$. Freedom from stroke 8 years after surgical intervention was $98.7 \% \pm 2.3 \%$ in patients who underwent a concomitant maze procedure compared with $88.7 \% \pm 2.6 \%$ in patients who underwent MVR alone $(P=.033$, Figure 4$)$. Three $(6.5 \%)$ of the 46 patients with an unsuccessful maze procedure had a stroke late after surgical intervention.

\section{Incidence of Bleeding After Surgical Intervention}

Seventy-six patients had major bleeding episodes after mechanical MVR. Twenty-three of those patients had intracranial bleeding, and the remaining 53 patients had extracranial bleeding. The INR at the time of either stroke or major bleeding is shown in Figure 5.

\section{Risk Factor for Late Stroke}

By means of univariate analysis, chronic postoperative atrial fibrillation (at 1 month after surgical intervention), omission of a maze procedure, early year of surgical intervention, and history of stroke were risk factors for late stroke (Table 5). Closure of the left atrial appendage was not a significant risk factor $(P=.69)$. By means of multivariate

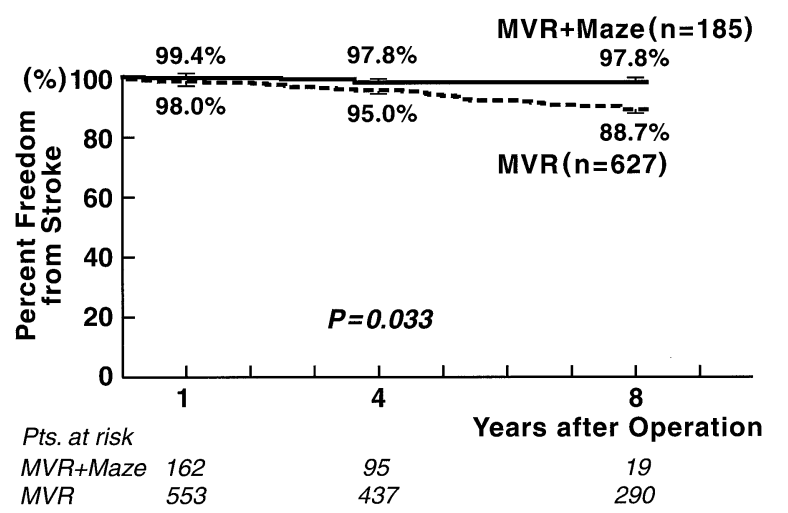

Figure 4. Freedom from stroke: effect of concomitant maze procedure. Bar indicates $\mathbf{9 5 \%}$ confidence interval.

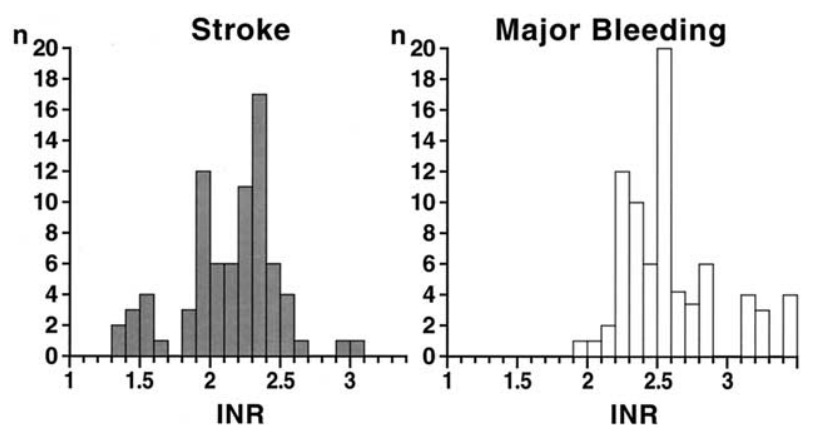

Figure 5. Effect of anticoagulation on stroke and major bleeding after mechanical MVR.

analysis, chronic atrial fibrillation and omission of the maze procedure were the 2 most significant predictors of late stroke (hazard ratio of 3.39 and 3.40, respectively), as determined by using multivariate analysis. Neither closure of the left atrial appendage nor left atrial plication had prevented late stroke.

\section{Discussion}

Although MVR with mechanical prostheses is a safe and commonly performed procedure, thromboembolic complications and bleeding related to the anticoagulant therapy remain significant causes of late morbidity and mortality. ${ }^{13}$ Recently, we and others reported the effect of the maze procedure on reducing the incidence of late stroke by restoring sinus rhythm. However, the risks for these complications and mortality after mechanical MVR were not fully described.

The current retrospective study confirms that mechanical MVR resulted in excellent 15-year survival (85\%, Figure 1). Furthermore, the only risk factors for late mortality were preoperative New York Heart Association class IV and older age ( $>65$ years, Table 4$)$. 
TABLE 5. Results of univariate and multivariate analysis for late stroke

\begin{tabular}{lccccc}
\hline & Univariate & & \multicolumn{3}{c}{ Multivariate } \\
\cline { 2 - 3 } \cline { 5 - 6 } Variable & $\boldsymbol{P}$ value & & $\boldsymbol{P}$ value & Hazard ratio & $\mathbf{9 5 \%} \mathbf{~ C ~}$ \\
\hline Chronic AF & $<.0001$ & & .0004 & 3.39 & $1.72-6.67$ \\
No Maze procedure & .003 & & .0279 & 3.40 & $1.14-10.14$ \\
Year of operation & .013 & & .0524 & 1.61 & $0.99-2.61$ \\
History of Stroke & .01 & & .0003 & 2.57 & $1.53-4.32$ \\
\hline
\end{tabular}

$\mathrm{Cl}$, Confidence interval; $A F$, atrial fibrillation.

This study also confirms that greater than $25 \%$ of patients with chronic atrial fibrillation had a stroke during the same 15 -year period (Figure 3). Because $78 \%$ of the patients who underwent MVR were in atrial fibrillation preoperatively (Table 1), restoration of sinus rhythm after surgical intervention was a critical factor in preventing strokes after mechanical MVR (Figure 3). The use of the maze procedure is a logical strategy to prevent late strokes after MVR with mechanical prostheses.

In the present study a maze procedure combined with MVR resulted in $98.7 \%$ freedom from stroke, whereas only $89 \%$ of the patients undergoing MVR alone were free from stroke 8 years after surgical intervention.

Moreover, chronic atrial fibrillation and the omission of a maze procedure were the 2 major predictors of late stroke after mechanical MVR. These results indicate that an adjunct maze procedure nearly eliminated the risk of late stroke after MVR with mechanical prostheses.

Among 185 patients who underwent a concomitant maze procedure, 3 patients had a stroke, but all of these patients were in atrial fibrillation at the time of their strokes. Moreover, none had left atrial contraction, which was confirmed by the absence of the a-wave on echocardiography. Patients with a failed maze procedure might have a risk for late stroke similar to that of patients with atrial fibrillation. The radial approach or new radiofrequency techniques preserve a more physiologic atrial transport function. ${ }^{14,15}$ Further study is necessary to elucidate the role of left atrial contraction in eliminating left atrial thrombus. ${ }^{16}$

In addition to maintaining sinus rhythm by using a maze procedure, closure of the left atrial appendage or reducing the size of an enlarged left atrium might also be expected to reduce the incidence of late stroke. In this study neither closure of the left atrial appendage nor plication of the left atrium prevented late stroke. However, more than $90 \%$ of patients with a combined maze procedure had the left atrial appendage closed, and the cutting and sewing in the original maze procedure could certainly have reduced the size of the left atrium. Differentiation of these factors might be difficult.

This study was also designed to examine the effect of anticoagulation on the incidence of late stroke. Among 72 patients who had a late stroke, 63 had an INR considered therapeutic (1.8-2.8) in the Japanese population (Figure 5). ${ }^{11,12}$ Thus therapeutic anticoagulation with warfarin alone is insufficient to prevent late strokes after mechanical MVR, especially in patients with chronic atrial fibrillation. This was a significantly lower target level compared with the American Heart Association/American College of Cardiology guidelines, ${ }^{7}$ the recommendations of the American College of Chest Physicians, ${ }^{17}$ and several European studies. ${ }^{18}$ However, recent studies from Japan indicate that maintaining an INR level between 1.8 and 2.8 results in lower bleeding and stroke rates compared with studies in the United States or Europe. ${ }^{11,12}$ Accordingly, the guidelines of the Japanese Circulation Society recommend a target INR of 1.8 to 3.0 for patients after MVR with bileaflet mechanical valves. ${ }^{19}$ The overall linearized risk of stroke in this study was only 0.02 per year in patients with atrial fibrillation and 0.004 per year in patients with regular rhythm. Moreover, the majority of patients who had major bleeding had INRs between 2.0 and 3.3 at the time of their events. Thus setting a higher INR might increase bleeding in our population. A prospective randomized study to determine the optimal anticoagulation regimen (eg, warfarin alone vs warfarin plus antiplatelet agents) is necessary. $3,8,18,20$

The major limitation of our study is that it was not randomized. The maze procedure was performed only after 1992, and therefore the follow-up period in the group undergoing MVR alone versus those undergoing MVR plus the maze procedure was different. The follow-up period for the group undergoing MVR plus the maze procedure (mean, 4.45 patient-years; range, 0.5-12.4 patient-years; total, 828 patient years) was significantly shorter compared with that of the group undergoing MVR alone (range, 0.5-22.9 patient-years; total, 5035 patient-years; $P<.0001$ ). Thus the significantly higher freedom from stroke in the maze procedure group might be related to a shorter observation period. However, our recent study regarding the impact of a maze procedure with mitral valve repair and replacement since 1992 indicated that the addition of a maze procedure significantly reduced the incidence of stroke between the groups during a comparable follow-up period. ${ }^{6}$ In addition to this, the decision for or against an adjunctive maze procedure reflected each surgeon's experience. Regarding anticoagulant therapy, the INR was known at the time of a stroke or major bleeding event, but baseline INR was only partly taken into account in this study. Thus the role of compliance as a cause of instability in oral anticoagulant therapy was not fully investigated. ${ }^{20}$

In conclusion, persistent atrial fibrillation was the most significant risk factor for early and late stroke after mechanical MVR. Restoration of sinus rhythm with the maze procedure nearly eliminated the risk of late stroke, whereas neither closure of the left atrial appendage nor therapeutic 
anticoagulation prevented this complication. Continuing efforts to define the optimal oral anticoagulant therapy to minimize the chances of stroke and bleeding are still necessary.

\section{References}

1. Hammermeister KE, Gulshan K, Henderson WG, et al. A Comparison of outcomes in men 11 years after heart-valve replacement with a mechanical valve or bioprosthesis. N Engl J Med. 1993;328:1289-96.

2. Fiore AC, Barner HB, Swarts MT, et al. Mitral valve replacement: randomized trial of St. Jude and Medtronic Hall Prostheses. Ann Thorac Surg. 1998;66:707-13.

3. Turpie AGG, Gent M, Laupacis A, et al. A comparison of aspirin with placebo in patients treated with warfarin after heart-valve replacement. N Engl J Med. 1993;329:524-9.

4. Cox JL, Ad N, Palazzo T. Impact of the maze procedure in the stroke rate in patients with atrial fibrillation. $J$ Thorac Cardiovasc Surg. 1999;118:833-40.

5. Handa N, Schaff H, Morris J, Anderson B, Kopecky SL, EnriquezSarano M. Outcome of valve repair and the Cox maze procedure for mitral regurgitation and associated atrial fibrillation. $J$ Thorac Cardiovasc Surg. 1999; 118:628-35.

6. Bando K, Kobayashi J, Kosakai Y, et al. Impact of Cox maze procedure on outcome in patients with atrial fibrillation and mitral valve disease. J Thorac Cardiovasc Surg. 2002;124:575-83.

7. Bonow RO, Carabello B, DeLeon AC, et al. ACC/AHA guidelines for the management of patients with valvular heart disease. J Am Coll Cardiol. 1998;32:1486-588.

8. Massel D, Little SH. Risks and benefits of adding anti-platelet therapy to warfarin among patients with prosthetic heart valves: a metaanalysis. J Am Coll Cardiol. 2001;37:569-78.

9. Kosakai Y. How I perform the maze procedure. Operative Techniques Thorac Cardiovasc Surg. 2000;5:23-45.

10. Rosendaal FR, Cannegieter SC, van der Meer FJM, Briet E. A method to determine the optimal intensity of oral anticoagulant therapy. Thromb Haemost. 1993;69:236-9.

11. Soga Y, Okabayashi H, Nishida T, et al. Up to 8-year follow-up of valve replacement with CarboMedics valve. Ann Thorac Surg. 2002; 73:272-9.

12. Matsuyama K, Matsumoto M, Sugita T, et al. Anticoagulant therapy in Japanese patients with mitral mechanical valves. Circ J. 2002;66:66870.

13. Akins CW. Results with mechanical cardiac valvular prostheses. Ann Thorac Surg. 1995;60:1836-44.

14. Nitta T, Lee R, Shcuessler RB, Boineau JP, Cox JL. Radial approach: a new concept in surgical treatment for atrial fibrillation I. Concept, anatomic and physiologic bases and development of a procedure. Ann Thorac Surg. 1999;67:27-35.

15. Williams MR, Stewart JR, Bolling SF, et al. Surgical treatment of atrial fibrillation using radiofrequency energy. Ann Thorac Surg. 2001; 71:1939-44.

16. Pasic M, Musci M, Siniawski H, et al. The Cox maze II procedure: parallel normalization of sinus node dysfunction, improvement of atrial function, and recovery of the cardiac autonomic nervous system. J Thorac Cardiovasc Surg. 1999;118:287-96.

17. Levine MN, Raskob G, Landefeld S, Kearon C. Hemorrhagic complications of anticoagulant treatment. Chest. 2001;119:108S-21S.

18. Cannegieter SC, Rosendaal FR, Wintzen AR, van der Meer FJM, Vanderbroucke JP, Briet E. Optimal oral anticoagulant therapy in patients with mechanical heart valves. $N$ Engl J Med. 1995;333:11-7.

19. Matsuda H, Abe T, Kawazoe K, et al. Guidelines for surgical and interventional treatment of valvular heart disease (JCS 2002). Circ J. 2002;66(suppl IV):1261-323.

20. van der Meer FJM, Briet E, Vanderbroucke JP, Sramek DI, Versluijs MHPM. The role of compliance as a cause of instability in oral anticoagulant therapy. Br J Haematol. 1997;98:893-900.

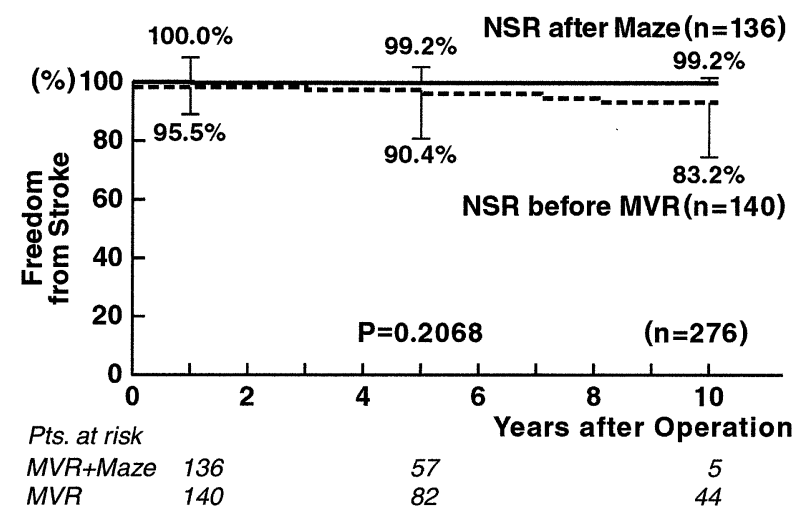

Appendix Figure 1. Freedom from stroke in patients with sinus rhythm after the maze procedure versus freedom from stroke in those in native sinus rhythm.

\section{Discussion}

Dr Cary Akins (Boston, Mass). I congratulate Dr Bando on this nice presentation of his continuing evaluation of the effect of concomitant maze procedures on the results of mitral valve surgery, which was similar to his presentation at this meeting a year ago. This year's presentation enlarges last year's study with the inclusion of about 40 patients undergoing the combination of the maze procedure and valve replacement and adds about 600 patients who had mechanical MVR from 1977 to 1992, none of whom had a maze procedure. This study concludes, as last year's study did, that sinus rhythm is associated with better long-term neurologic results but that sinus rhythm apparently has no demonstrable effect on long-term survival.

I have several questions about the study design, the first concerning the mechanical prostheses implanted because we know that different prostheses have different thrombogenic potentials. Did all of the patients in this study going back to 1977 receive the same mechanical prosthesis? If different prostheses were used, was prosthesis type inserted as a variable in the Cox analysis?

Dr Bando. Thank you very much, Dr Akins, for your excellent questions. Regarding the type of valves, among these 812 patients, 533 patients had a St Jude mechanical valve, and the remaining valves include the CarboMedics valve in 125 patients and, recently, the ATS valve in 47 patients. Earlier in our experience, the Björk-Shiley valve was used in 70 patients, and the Omniscience valve was used in 23 patients. We did analyze the type of valves as potential predictors in univariate and multivariate analyses, and these were entered into a Cox proportional hazards model, but none of the valve types came out as a risk factor for either late mortality or late stroke.

Dr Akins. My second question focuses on the effect of obliteration of the left atrial appendage on late stroke. The authors include in their statistical analysis of this part of the study patients who had a concomitant maze procedure. Wouldn't it be better to eliminate the maze patients and analyze only patients who had a MVR with or without obliteration of the left atrial appendage?

Dr Bando. That is a very excellent point, Dr Akins. Removing 185 patients, we have 627 patients undergoing MVR alone, and if 